Most patients (77\%) and general practitioners $(63 \%)$ were dissatisfied with the new contract exclusion criteria; $23 \%$ of patients and $36 \%$ of general practitioners were satisfied with it. In all, $51 \%$ of general practitioners re-referred their patient either to another hospital $(36 \%)$ or back to the original unit $(15 \%)$.

\section{Comment}

There is low acceptance among general practitioners and patients of recently imposed contract exclusions. Subsequent re-referrals transfer patients from one waiting list to another, wasting NHS resources without making waiting lists shorter. It is worrying that one patient with a malignancy was refused NHS treatment under the new system, and this error is consistent with the findings of previous research suggesting that it is unreasonable to expect general practitioners to have the diagnostic and therapeutic skills of specialist clinicians. ${ }^{1}$ These findings raise questions about the general practitioner's role in diagnosis and management in the context of restricted secondary services.

Rationing of health services is emotive, involving issues of ethics, finance, and standards of care. ${ }^{23}$ Although possibly a legitimate factor in modern healthcare provision, rationing may be unacceptably restrictive to patients and clinicians. In Florida in 1996, Medicare's decision to restrict funding of excision of actinic keratoses resulted in litigation (although unsuccessful) by the American Academy of Dermatology, the Florida Society of Dermatology, and the Seniors Coalition. ${ }^{4}$

With rising political and financial pressures to cut NHS waiting lists, patients with urgent problems are given priority. Those with apparently benign conditions may wait for years to be seen in clinics, or they could be excluded from waiting lists altogether. ${ }^{5}$ If similar restrictions on service provision continue, this could constitute implicit acknowledgement of an "acceptable level" of missed malignancy. Although rationing of services may be necessary, care must be exercised in its application.

Contributors: CO'B conducted the study and collated the data The paper was written jointly by $\mathrm{CO}^{\prime} \mathrm{B}$ and $\mathrm{RC}$. RC is the guarantor.

Funding: Plastic surgery department, Salisbury Health Care NHS Trust.

Competing interests: None declared.

1 McWilliams LJ, Knox F, Wilkinson N, Oogarah P. Performance of skin biopsies by general practitioners. BMJ 1991;303:1177-9.

Latham SR. The ethics of managed care: financial incentives to limit care. Clin Plast Surg 1999;2:115-21.

3 Asch DA, Ubel PA. Rationing by any other name. $N$ Engl $J$ Med 1997;336:1668-71.

4 Charatan FB. Skin lesion removal rationed in Florida. BMJ 1996;313 1506

5 Timmons TJ. Rationing of surgery in the National Health Service: the plastic surgery model. Ann R Coll Surg Engl 2000;82(suppl):332-3.

(Accepted 10 April 2001)

\title{
Cohort survey of heart valve replacement patients: does the valve card scheme have room for improvement?
}

\author{
Maria-Benedicta Edwards, Kenneth M Taylor
}

Patients undergoing heart valve replacement have a high risk of experiencing valve related adverse events such as acute failure of the valve mechanism, valve thrombosis, and peripheral emboli. Immediate availability of information about valves for any valve patient presenting at outpatient clinics, general practitioner surgeries, or acutely at accident and emergency departments may lead to early suspicion and diagnosis of specific complications known to be associated with particular heart valves. ${ }^{1-3}$ Postoperative surveillance is therefore crucial.

Valve manufacturers have established schemes to provide patients with cards that give details of the valve prosthesis. The process has four to five steps and can fail at any stage, for example when the implanting centre sends implant details to the valve manufacturer (usually via the UK distributor), or when the valve manufacturer sends the implant card to the patient via the implanting centre.

Clinicians have been concerned that current distribution systems may be less than optimal; although valve card schemes have been in place for many years, they have not been independently evaluated. We surveyed a cohort of UK patients who had had heart valves replaced to determine what proportion received an implant card, and when they received it relative to their implant date.

\section{Methods and results}

The UK heart valve registry prospectively collects data on all valve replacements carried out in the United Kingdom. ${ }^{4}$ Currently $>79000$ patients and $>88000$ heart valves are registered. Patients who underwent heart valve replacement between 1 January 1998 and 31 December 1998 were identified from the database and checked against national registers (England, Scotland, Wales, and Northern Ireland) for deceased patients and those lost to follow up. The 2582 patients identified were sent an anonymised questionnaire and asked the date of their operation, if they had received an implant card, and the interval between operation and card receipt.

A total of 1914 patients (74\%) responded to the questionnaire. Of these, 47\% $(n=898)$ had not received an implant card 12 months postoperatively (figure). In all, 14\% had received their implant card one month postoperatively, $30 \%$ three months postoperatively, and $43 \%$ six months postoperatively. A small number of patients $(\leqslant 1 \%)$ could not remember how long after surgery the card had arrived. $45 \%$ identified themselves and were matched against valve manufacturer and implanting centre; $60 \%$ of these $(n=519)$ did not receive an implant card. Three of nine valve manufacturers achieved $>50 \%$ (range $53-71 \%$ ) success in getting implant cards to patients within a year post-
United Kingdom Heart Valve

Registry,

Department of Cardiothoracic Surgery,

Hammersmith

Hospital, London W12 0NN

Maria-Benedicta

Edwards

manager

Kenneth M Taylor professor

Correspondence to: M-B Edwards m.b.edwards@ic. ac.uk

BMJ 2001;323:429-30

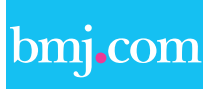

A list of participating hospitals is available on the $B M J$ 's website 


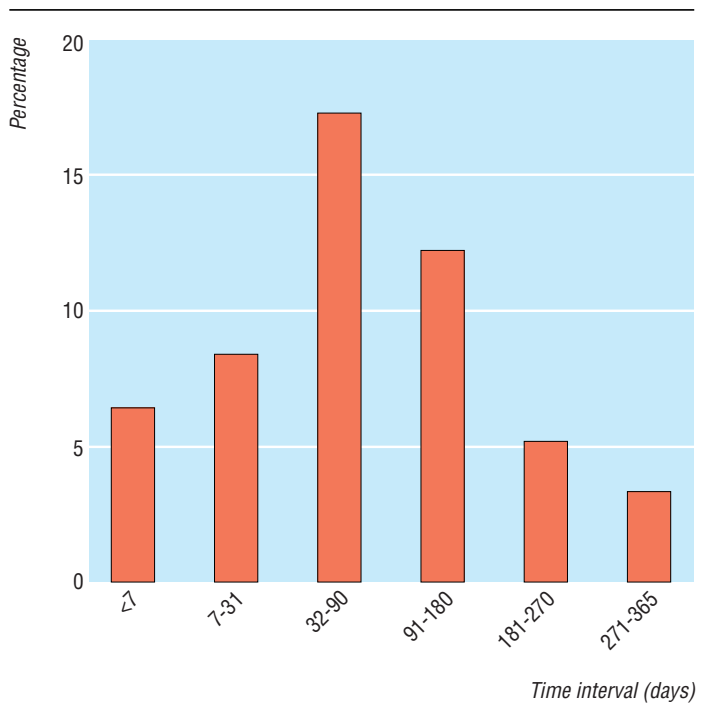

Implant cards received by patients over time

operatively; the remainder achieved $0-45 \%$ success. Although certain patients were identified against their individual implanting centre, it seems that some implanting centres are better than others at forwarding implant cards to patients.

\section{Comment}

Present implant card schemes are falling well below acceptable standards. Nearly half of the patients sampled had not received an implant card 12 months postoperatively. Furthermore, patients who had not received the implant card by six months postoperatively were unlikely ever to do so. It would be unfair, however, to blame valve manufacturers alone. Implanting hospitals may fail to send details to the manufacturer or the card to the patient.

We believe that the situation could easily be improved. Currently, UK cardiac centres send implant details to the heart valve registry. Authorising the registry to send the implant card to the patient would remove the manufacturer and clinician from the process. The manufacturer would receive anonymised implant information for postmarket surveillance and patients would receive their cards quickly. This centralised system would be cost effective, could be financed through a small charge per valve implanted, and would contribute to patient surveillance.

We are grateful to all staff involved with the UK heart valve registry for assimilating and maintaining the national database. All participating hospitals do so voluntarily (see BMJ's website for a list of hospitals involved).

Contributors: M-BE designed the study and wrote the paper. She is also the guarantor. KMT is director of the UK heart valve registry and helped write the paper.

Funding: None.

Competing interests: None declared.

1 Baumgartner FJ, Munro AI, Jamieson WRE. Fracture embolization of a Duromedics mitral prosthesis. Tex Heart Inst J 1997;24:122-4.

2 Dyer C. Six deaths prompt alert over artificial heart valve. Guardian 1988 Sept 17:20.

3 Van der Graff Y, De Waard F, van Herwerden LA, Defauw J. Risk of strut fracture of Bjork-Shiley valves. Lancet 1992;339:257-61

4 Edwards MB, Taylor KM. A profile of valve replacement surgery in the UK (1986-1997): a study from the UK Heart Valve Registry. J Heart Valve Disease 1999;8:697-701.

5 Taylor KM. The United Kingdom Heart Valve Registry: the first 10 years [editorial]. Heart 1997;77:295-6.

(Accepted 28 March 2001)
Oaktree Cottage, Mitford, Morpeth, Northumberland NE61 3PN

Gordon Pledger consultant in public health medicine (retired)

Department of Paediatric Surgery, Leeds Teaching Hospitals NHS Trust, Leeds LS9 7TF

Mark D Stringer consultant paediatric surgeon

Correspondence to: G Pledger gordonpledger@ yahoo.co.uk
The number of children under 15 years of age certified as dying of acute appendicitis each year in England and Wales decreased from the 1930 s to the 1980 s. Factors contributing to death have been analysed in audits in 1963-7 and 1980-4. ${ }^{2}$ We undertook an audit of children dying in 1993-7, compared these results with those of previous audits, examined hospital administrative statistics, ${ }^{3}$ and identified possible reasons for the fall in the number of deaths.

\section{Methods and results}

We used similar methods to previous audits to examine hospital and coroner's reports of all 12 childhood deaths in 1993-7. ${ }^{12}$ The median age of the children who died was 10.6 (range 3.0-14.2) years. Six children died at home or on arrival at hospital, and six died postoperatively in hospital from multiorgan failure (four in district general hospitals, two in children's hospitals). Median duration of symptoms before hospital admission or death was 3 (2-6) days. Difficulty or delay in diagnosis was the main factor contributing to death in six cases.

The number of deaths from acute appendicitis in hospital fell from an annual average of 36.2 in 1963-7 to 1.8 in $1993-7$. The case fatality rate in hospital has fallen from 1.06 to 0.16 per 1000 discharges with acute appendicitis, a fall of $85 \%$ (table). If children who were moribund on arrival at hospital are excluded the decrease is $97 \%$. If the $1963-7$ age specific death rates had applied to children admitted with acute appendicitis in 1993-7 the expected number of deaths would have been 50 rather than the nine recorded. Thus 41 of 172 (24\%) fewer deaths in 1993-97 may be attributed to a fall in the hospital case fatality rate, the greatest improvement being in the youngest age group.

The number of children with a discharge diagnosis of "acute appendicitis" fell from an annual average of 